\title{
Evaluation of Sirt1 And Foxo's In Primary Tumor And Distant Organ Metastasis Invaded By Benign And Highly Metastatic Breast Carcinoma Cells Under In Vitro And In Vivo Conditions.
}

\section{Sayra Dilmac}

Akdeniz University School of Medicine

Nilay Kuscu

Akdeniz University School of Medicine

Ayse Caner

Egerton University School of Medicine

Sendegul Yildirim

Akdeniz University School of Medicine

\section{Burcak Yoldas}

Akdeniz University School of Medicine

Gamze Tanriover ( $\square$ gamzetanriover@akdeniz.edu.tr)

Akdeniz University School of Medicine

Research article

Keywords: SIRT1, FoxO, primary tumors, metastasis, breast cancer

Posted Date: February 8th, 2021

DOl: https://doi.org/10.21203/rs.3.rs-183690/v1

License: (c) (i) This work is licensed under a Creative Commons Attribution 4.0 International License.

Read Full License 


\section{Abstract}

Breast cancer is the second most common cancer in women. In malignant breast cancers, tumor cells have the potential to metastasize to distant organs through the lymphatic system and blood circulation. The aim of this study is to evaluate the expression of SIRT1 and FoxO proteins in metastatic and nonmetastatic breast cancer cells and distant organs metastasis.

In our study, SIRT1, p53, p21, and FoxO proteins have been evaluated in metastatic 4TLM and nonmetastatic 67NR cell lines by immunocytochemistry in vitro and also in mice breast cancer model in vivo. Cells were orthotopically injected to mammary fat pads of 8-10 weeks old Balb/c female mice. Primary tumor, lung and liver tissues were removed and expressions of these proteins were evaluated by immunohistochemistry, western blot and RT-PCR. In addition, signal pathways that are related to SIRT and FoxO proteins were examined by using IPA core analysis. TCGA database was browsed for investigation of different genes.

In primary tumors, SIRT1, p21, p53, E2F1 and FoxO expressions were higher in 67NR compared to 4TLM. In metastatic lung and liver tissues, the expression levels of SIRT1, Fox01, FoxO3a and FoxO4 proteins were increased in 4TLM compared to 67NR. IPA and TCGA analysis have also revealed that SIRT1 and FoxO proteins are lower in primary tumors, but increased in metastatic stages.

In conclusion, in primary tumors SIRT1 and FoxO expressions were decreased in 4TLM compared to 67NR. Moreover, SIRT1 and FoxO, especially expressed in metastatic cells. High level of FoxO expressions in metastatic stages in TNBC patients also supports its association with metastasis. Our findings suggest that SIRT1 and FoxO's have crucial role in tumor progression metastatic process in breast cancer.

\section{Introduction}

Breast cancer is most frequently type of cancer which is diagnosed in woman and most common cause of cancer-related death in world-wide [1,2]. The overall average 5-year survival rate for patients with early breast cancer is high as compared to the patients who have developed very longer than those with distant metastasis. The main reason of breast cancer related deaths is not primary tumors but metastatic spread to the distant organs [3]. Although metastasis is the major cause of treatment failure in cancer patients, metastatic process and inhibition of metastasis have not been fully clarified yet.

Mutation of either proto-oncogenes and tumor suppressor genes (TSG) could trigger tumorigenesis and cancer metastasis. Proto-oncogenes are involved in the regulation of the cell cycle, but overexpression of proto-oncogenes could also lead to uncontrolled cell division [4]. TSGs are known as negative regulators of tumor growth by controlling cell division, however, their down regulation can also increase proliferation and the metastatic ability of cancerous cells [5]. Therefore, mechanistic role these genes and/or its related pathways are needs to be clarified in tumorigenesis and metastasis. 
Sirtuins (SIRT) (Silent information regulator), NAD (Nicotinamide adenine dinucleotide) -positivedependent class III histone deacetylases (HDACs) [6], is a novel oncogene [7]. SIRT1 plays an important role in cell survival process by regulating the transcriptional activities of p53 and by inducing apoptosis $[8,9]$ and by suppressing FoxO proteins $[10,11]$. It has been shown that overexpression of SIRT1 could induce tumor growth and increases cell survival ability in cancer cells [9].

The forkhead box $\mathrm{O}$ (FoxO) family of transcriptional factors comprises of 4 members: FoxO1, FoxO3, FoxO4 and Fox06 [12]. FoxO proteins are "double-edge swords" dualistically involved in the regulation of various steps of carcinogenesis and metastasis. FoxO transcription factors are involved in various crucial mechanisms, such as apoptosis, cell cycle arrest, resistance to oxidative stress, DNA repair mechanism, glucose metabolism, energy, homeostasis and cellular differentiation [13]. FoxO proteins are regulated by acetylation and phosphorylation-dependent ubiquitination mechanisms [14]. Oxidative stress causes the transactivation of FoxO by catalyzing its deacetylation in an NAD-dependent manner regulated by SIRT1 [15]. Under stress conditions such as, apoptosis and DNA repair regulation, SIRT1 forms a complex in the nucleus and deacetylates the FoxO proteins and affects FoxO1 by reducing stress [10]. If FoxO is inhibited, the gene transcription required in apoptosis steps cannot be induced either [16]. FoxO4 induced apoptosis in SIRT1-silenced HCT116 cancer cells [17]. MRP2 (multidrug resistance-associated protein 2) has been shown to be transcriptionally upregulated by Fox01 in tamoxifen-resistant breast cancer cells [18]. Taken together, this finding suggests that the interaction between SIRT1 and Fox01 may play an effective role in metastasis process and treatment of breast cancer. Hu Q. et al. showed that on bladder cancer that inhibition of SIRT1 increased FoxO3a and acetylated FoxO3a. Increased FoxO3a acetylation affected cell cycle regulation and antioxidant response [19]. In the knockdown study performed with PANC1-SIRT1-RNAi cells, it was observed that FoxO3a expression increased significantly but it did not affect p53 expression [20] However, the effects of SIRT1 and FoxOs on breast cancer metastasis have not been explained yet.

Mutations in the p53 gene, which regulates cell cycle and apoptosis, cause loss of tumor suppressor functions, providing in tumor progression [21]. According to clinical evidence, more than $50 \%$ of human cancers have been found to be related to p53 mutation [22]. SIRT1-p53 axis plays a complex role in tumorigenesis with dual functions in tumor-promotion and tumor suppression [23, 24]. p21 (CDK1), is also known a cyclin dependent kinase inhibitor [25]. p21 is responsible for the regulation of the cell cycle in $\mathrm{G} 1$ and $\mathrm{S}$ phase, and its expression is controlled by the p53 protein [26]. Although, p53 promotes apoptosis by up-regulating p21, [25] and mutant p53 induces cell proliferation and survival via decreasing p21 [27]. FoxOs and p53 share several downstream target genes, including p21 [28], it suggest that FoxOs and p53 may also co-regulate tumor suppressor signaling.

E2F, another gene family that also plays critical roles in cell cycle regulation, consists of 8 different genes [29]. E2F1 (E2F Transcription Factor 1) is a well-known member in this family [30]. E2F1 is a major Rb (retinoblastoma)-binding protein [31] and also is an important for G1-S transition of cell cycle [30]. E2F1 promotes p53-mediated apoptosis by inducing the expressions of apoptosis-related proteins [32, 33]. Lee J. S. et al. have shown that high expression of E2F1 promotes metastasis in bladder cancer [34]. In 
addition, decreased expression of E2F1 and apoptosis-related genes has been shown to adversely affect patient survival in breast and ovarian cancer patients [35]. In metastatic melanoma showed that inhibition of E2F1 reduced the migration and invasiveness of melanoma cancer cells, but had no effect on cell proliferation [36]. Hollern D.P. et al. showed that the loss of E2F1 promotes metastasis by rearranging Fgf13 (Fibroblast growth factor 13) in breast cancer cells [37]. Considering these studies, E2F1 appears to play a bidirectional role in cell survival.

Based on the literature information and findings regarding to the roles of SIRT1 and FoxO in tumor growth and apoptosis, and their possible roles on metastasis, the aim of the present study is to investigate the effect of SIRT1 and FoxO and its related pathways on tumor growth and metastasis in primary tumors and distant organs by using both benign and highly metastatic breast cancer cells under in vitro and in vivo conditions.

\section{Material And Methods}

\subsection{In vitro Experimental Procedures 2.1.1. Cell Culture}

4T1 cells were previously derived from spontaneously formed breast tumors in Balb/c mice as previously described [38]. The 4THM (4T1-Heart Metastasis) cell line was derived from cardiac metastasis of 4T1 cells which are created by Erin N. et al. [39]. These 4THM cells were implanted orthotopically into Balb/c mice, thereby establishing macroscopic liver metastasis, which in turn were used to develop an additional cell line, designated as 4TLM (4T1- Liver Metastasis) [40]. Also, non-metastatic 67NR and metastatic 4TLM cell lines were grown in DMEM-F12 (Dulbecco's Modified Eagle Medium/Nutrient Mixture F-12) (Invitrogen; \#11320074, MA, USA) supplemented with 5\% FBS (Fetal bovine serum) (Invitrogen; \#10270106, MA, USA), 2 mM L-glutamine (Invitrogen; \#25030024, MA, USA), 1 mM sodium pyruvate (Invitrogen; \#11360039, MA, USA), and 0.02 mM non-essential amino acids (Invitrogen; \#11140035, MA, USA).

\subsubsection{Immunocytochemistry}

The expression of SIRT1, p53, p21 and FoxO proteins in 4TLM and 67NR cells were evaluated by using immunocytochemistry. Firstly, cells were fixed in 4\% paraformaldehyde (Merck; 1.04005.1000, MA, USA) and washed with Phosphate-buffered saline (PBS), blocked with blocking solution 1,5 g Bovine Serum Albumin (Euroclone; EMR086025, Pero, MI), 0,0375 Glycine (Bio-Rad; \#161-0718, CA, USA). Then cells incubated with anti-SIRT1 (Santa Cruz; \#sc-15404, CA, USA, 1/100 dilution), anti-Fox01 (Cell Signaling; \#2880S, MA, USA 1/100 dilution), anti-FoxO3a (Cell Signaling; \#12829S, MA, USA 1/100 dilution), and anti-FoxO4 (Santa Cruz; \#sc-25539, CA, USA 1/100 dilution) for 2 hours at room temperature. After the incubation, the primary antibodies were removed from the coverslip and cells were incubated with the secondary antibody Alexa Fluor 555 and Alexa Fluor 488 (Thermo Fisher; \#A11008, MA, USA 1/250 
dilution) for 1 hour at room temperature. The mounting medium containing DAPI (Vector Labs; \#H-1200, CA, USA) was dropped and slides were examined under fluorescence microscope.

To reveal which pathways SIRT1 can use in the cell cycle, the expression of p53 (Santa Cruz; \#sc-6243, CA, USA 1/100 dilution) and p21 (Santa Cruz; \#sc-756, CA, USA 1/100 dilution) proteins in both benign and highly metastatic tumor cells were also evaluated.

\subsection{In vivo Animal Studies}

\subsubsection{Animal Models}

Female Balb/c mice were obtained from Kobay Animal Laboratory (Ankara, Turkey) and kept under a $12 \mathrm{~h}$ light-dark cycle and a controlled diet. All experimental protocols were approved by the Local Ethics Committee for Animal Research (2016.09.02).

4TLM and 67NR cells in HBSS (Hanks' Balanced Salt Solution) (Sigma Aldrich; \#H9269, GE) (for 4TLM $1 \times 10^{5}$ cells each mouse; for $67 N R 1 \times 10^{6}$ cells each mouse) were injected into the right upper mammary fat pad just beneath the armpit of BALB/c mice under ketamine/xylazine anesthesia ( $15 \mathrm{mg} / \mathrm{kg}$ i.m.). Formation of $67 \mathrm{NR}$ tumors requires implantation of higher number of cells. For each group 8 animals were used.

\subsubsection{Immunohistochemistry}

Primary breast tumors, lung and liver tissues were removed 27 days after injection of tumor cells. They were fixed with $10 \%$ formaldehyde (Merck; 1.04003 .1000$, MA, USA) and embedded in paraffin. Five $\mu \mathrm{m}$ thick sections of the paraffin blocks were taken to the positively charged slides.

Paraffin sections were deparaffınized, rehydrated and blocked for endogenous peroxidase activity with methanol (Merck; \#1.060.092.511, MA, USA) containing 3\% $\mathrm{H}_{2} \mathrm{O}_{2}$ (Merck; \#1.08600.1000, MA, USA) for 15 min. After the incubation with $\mathrm{H}_{2} \mathrm{O}_{2}$ were used universal blocking reagent (Thermo Fisher; \#TA-125-UB, MA, USA) for blocking nonspecific binding, during 7 min at RT. Anti-SIRT1 (Santa Cruz; \#sc-15404, CA, USA 1/200 dilution), anti-p21 (Santa Cruz; \#sc-756, CA, USA 1/200 dilution), anti-p53 (Santa Cruz; \#sc6243, CA, USA 1/200 dilution), anti-E2F1 (Abcam; \#ab-179445, MA, USA 1/100 dilution), anti-FoxO1 (Cell Signaling; \#2880S, MA, USA, 1/100 dilution), anti-FoxO3a (Cell Signaling; \#21829S, 1/100 dilution), antiFoxO4 (Cell Signaling; \#2359, MA, USA, 1/100 dilution), and anti-Cleaved caspase 3 (Cell signaling; \#9661L, MA, USA 1/50 dilution) antibodies diluted in dilution buffer were applied for overnight at $+4^{0} \mathrm{C}$ in a humidified chamber. After several washes in PBS, sections were incubated for $1 \mathrm{~h}$ at room temperature with biotinylated goat anti-rabbit IgG secondary antibody (Vector Lab; \#BA1000, CA, USA, 1/400 dilution). Following washing steps with PBS, sections were incubated by using HRP streptavidin-peroxidase complex (Invitrogen; \#85-9043, MA, USA) for $20 \mathrm{~min}$ at room temperature. Antibody-antigen complexes were visualized by incubation of 3,3'-diaminobenzidine (DAB) (Sigma Aldrich; \#D4168, GE) chromogen. Sections were counterstained with Mayer's hematoxylin (Merck; \#1.09249.1000, MA, USA), dehydrated 
and coverslips with entellan (Merck; \#1.0791.0500, MA, USA). All sections examined under Zeiss-Axioplan (Carl Zeiss $\mathrm{GmbH}$, Jena, Germany) microscope.

\subsection{Image J Analysis of Immunohistochemical Staining}

The micrographs of tissue samples from all groups were taken using SPOT Advanced 4.6 at 10x and 40x magnification micrographs. All these micrographs were analyzed by using Image 1.46 (Image Processing and Analysis in Java; US National Institutes of Health, Bethesda, MD; https://imagej.nih.gov/ij/) software by scanning 10 non-overlapping fields in each issue and expressing the positive areas as a percentage of the total area.

\subsection{Western Blotting}

For Western-blot analysis, total proteins from the primary tumor tissues were extracted in the lysis buffer (10 ml 0.1 M Tris (Merck; \#1.08387.0500, MA, USA), 0,184 g Sodium orthovanadate (Sigma Aldrich; S6508, GE) and the protease inhibitor cocktail (Roche; \#1169749800120, CH). The protein concentrations were calculated by using the Bradford Protein Assay kit (Biorad; \#1-800-4, CA, USA). For each group, $75 \mu \mathrm{g}$ protein was loaded into each well, separated by SDS-polyacrylamide gel electrophoresis using $10 \%$ TRIS-HCl gels, for SIRT1, p53, p21, E2F1, Fox01 and FoxO3a antibodies and GAPDH for internal control. The proteins were blotted onto poly vinylidene fluoride (PVDF) membrane (Thermo Fisher; \#88520, MA, USA). Membranes were washed twice with TBS-T (0.05\% Tween 20 in 10x TBS) and then blocked with $5 \%$ Blotting-Grade Blocker nonfat dry milk (Biorad; 1706404xtu, CA, USA) in TBS-T for $1 \mathrm{~h}$ at room temperature. Afterwards, the membranes were incubated with anti-SIRT1 (Santa Cruz; \#sc-15404, CA, USA, 1/1000 dilution), anti-p21 (Santa Cruz; \#sc-756, CA, USA, 1/1000 dilution), anti-p53 (Santa Cruz; \#sc-6243, CA, USA, 1/1000 dilution), anti-E2F1 (Abcam; \#ab-179445, MA, USA, 1/1000 dilution), antiFox01 (Cell signaling; \#2880S, MA, USA, 1/1000 dilution), anti-FoxO3a (Cell signaling; \#21829S, MA, USA, 1/1000 dilution) and GAPDH (Cell signaling; \#5174S, MA, USA, 1/2000 dilution) primary antibodies at $4^{\circ} \mathrm{C}$ overnight. Following the washing step with TBS-T, all membranes were incubated with a secondary antibody with peroxidase labeled anti-rabbit IgG (Vector; \#PI-1000, CA, USA, 1/4000) at room temperature for $1 \mathrm{~h}$. The immunoblots were developed using an ECL Kit (Thermo Fisher; \#34080, MA, USA) and subsequently, membranes were exposed to Bio Max film (Kodak; \#05-728-26, MA, USA).

\subsection{Real Time-PCR}

Total RNA was extracted from primary tumor samples using RNeasy Mini Kit, (Qiagen; \#74104, NL) according to the manufacturer's protocol. A nanodrop spectrophotometer (Thermo Fisher; MultiscanGo, MA, USA) was used to measure absorbance at A260/280 and A260/230 to assess RNA concentration and quantity. cDNA was obtained by reverse transcriptase PCR method, which was true to the commercial kit protocol (EvoScript Universal cDNA Master Kit, Qiagen; \#7912374001, NL). Gene expression analysis was performed with the obtained cDNAs by following the commercial kit protocol (FastStart Essential DNA Green Master, Qiagen; \#6402712001, NL). The primers used in RT-PCR were designed with sequences based on the references from literature [41, 42] (Table 1). The Ct value of the fluorescence radiation values measured on the instrument by means of SYBR Green probe was averaged over 3 
technical repetitions. It was normalized with ribosomal 18 s (housekeeping) used as reference gene. All genes were normalized with housekeeping gene and $\Delta \Delta \mathrm{Ct}$ (= Fold Change: fold change in mRNA expression levels) was calculated using the arithmetic formula for comparative quantification.

\subsection{Bioinformatics Analysis}

Microarray data of gene expression profiles belonged to GSE62598 data set was downloaded from Gene Expression Omnibus (GEO) (www.ncbi.nlm.nih.gov/geo/) with based on the platform GPL7202 (Agilent014868 Whole Mouse Genome Microarray). Gene expression profiling in this data set could reveal distinct expression patterns associated with 4T1 subpopulations derived from breast tissues, primary breast tumor, and liver and lung metastatic tumor. The dataset contained 3 tissue samples each group. All data were processed using the R software (www.r-project.org). For GEO data, the limma package was used for identifying differentially expressed genes (DEGs) between the primary/metastasis samples and breast tissue samples [43]. An adjust $\mathrm{P}<0.05$ and the absolute log2 fold change $(\log 2 \mathrm{FC})>1$ was considered statistically significant. To analyses the potential biological processes, and pathway of the overlapping DEGs, Ingenuity Pathway Analysis (IPA, www.qiagen.com/ingenuity) was performed with $\mathrm{P}<0.01$ and absolute $\log 2 \mathrm{FC}>1$ as the threshold values. The RNA sequencing datasets and matched clinicopathological information of Breast Invasive Carcinoma (BRCA) were downloaded from TCGA database (https:// tcga-data.nci.nih.gov/). The mRNA expression of FoxO family members and SIRT1 were analyzed in TNBC and normal tissue by the Gene Expression Profiling Interactive Analysis Platform [44]. Also, levels of these gene expressions were investigated in non-metastasis and metastasis situations of TNBC.

\subsection{Statistical Analysis}

Statistical significance was determined by analysis of variance with the Dunnett posttest by Graph Pad Prism 8 (San Diego, CA, USA) software. Data are shown as mean \pm standard error of the mean and were considered statistically significant at $p<0,0001$.

\section{Results}

\subsection{Immunofluorescence findings: SIRT1, p53, p21 and FoxOs expressions in both 67NR and 4TLM tumor cells}

The expressions of SIRT1, p53, p21 and FoxO proteins were determined in both benign (67NR) and malign (4TLM) breast cancer tumor cells. The expression of SIRT1 protein is limited in nucleus and its expression was higher in 67NR cells compared to 4TLM (Fig. 1a). On the contrary, cytoplasmic expression of p53 in 67NR was nuclear in 4TLM (Fig. 1a). p21 has found to localized in nucleus in both 67NR and 4TLM cells but its expression levels were decreased in 4TLM cells compared to 67NR (Fig. 1a). The presence of p53 and p21 proteins in cytoplasm in tumor cells, is important for the control of the cell cycle. Furthermore, Fox01, FoxO3a and FoxO4 expressions were observed in cytoplasm of the 4TLM and 
67NR cell lines (Fig. 1b). While Fox01 expression was higher in 4TLM cells compared to 67NR, Fox03 and FoxO4 expressions were higher in 67NR cells compared to 4TLM.

\subsection{Immunohistochemistry findings: SIRT1, p53, p21, E2F1, cleaved caspase 3 and FoxOs expressions in both 67 NR and 4TLM primary tumors and distant metastatic organs}

\subsubsection{Primary Tumors}

Expression levels of SIRT1 and FoxO proteins in primary tumors were evaluated to reveal the differences of these proteins in metastatic and non-metastatic tumors. In order to reveal the relationship between metastatic potential of tumor and cell viability, p53, p21, E2F1 and cleaved caspase 3 proteins were evaluated. The expression level of SIRT1 was decreased significantly in 4TLM primary tumors compared

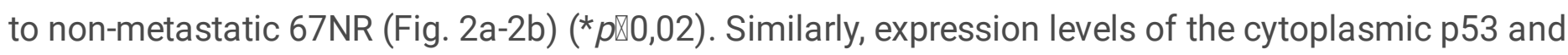
p21 expressions were decreased significantly in metastatic 4TLM primary tumors when compared to nonmetastatic 67NR (Fig. 2a-2b) ( ${ }^{\star * \star *} \mathrm{p} \otimes 0,0001$ ). In the 67NR primary tumors, E2F1 is located in the nucleus and its expressions was higher compared to 4TLM, whereas, in 4TLM tumors E2F1 showed perinuclear localization and its expressions was lower significantly compared 67NR (Fig. 2a-2b) (** $p \llbracket 0,001)$.

All Fox01, FoxO3a, and FoxO4 proteins were strongly expressed in 67NR (Fig. 2c-2d). However, their

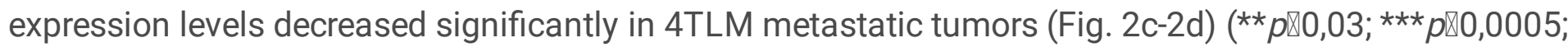
$\star \star \star \star p \otimes 0,0001)$, indicating that the lack of 4TLM FoxO proteins could be associated the metastatic process. As it is known in the literature, increased FoxO expression decrease metastasis; it was thought that low FoxO expression in primary tumors may also trigger metastasis. Cleaved caspase 3 , a marker of apoptosis, increased in metastatic 4TLM primary tumors especially in necrotic cells when compared to

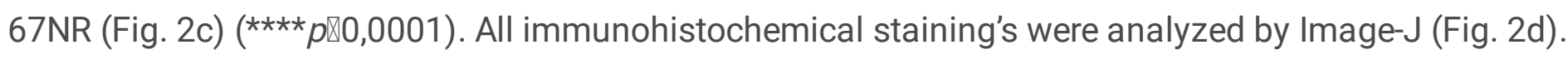

\subsubsection{Metastatic Tissues}

The expression levels of SIRT1, p53, p21, E2F1, cleaved caspase 3 and FoxO proteins were evaluated in metastatic liver and lung tissues.

\subsubsection{Liver}

The expression of SIRT1 was increased in 4TLM metastatic liver tissue compared to 67NR nonmetastatic liver tissue and tumor free liver tissue. Nuclear and cytoplasmic SIRT1 expression was increased especially metastatic areas of liver tissues. The expression was observed in infiltrative cells around the hepatic artery in metastatic microenvironment (Fig. 3a-3b). Cytoplasmic SIRT1 expression was observed in hepatocytes in non-metastatic liver tissue. In addition, p53 expression was also increased in 4TLM groups. Similar to SIRT1, nuclear p53 expression was observed in infiltrative cells around the hepatic artery. (Fig. 3a). Expression of p21 was cytoplasmic in some Kupffer cells in the tumor free liver tissue. (Fig. 3a). E2F1 expression in 4TLM and 67NR liver tissue was observed on perinuclear staining in hepatocytes. In addition, E2F1 expression was intensely observed in infiltrative cells in the 
4TLM group. E2F1 expression in the livers of the 4TLM group was observed cytoplasmic in Kupffer cells. (Fig. 3a-3b). E2F1 expression was lower in the tumor free group liver, while its increased expression in the 4TLM and 67NR groups compared to tumor free group. (Fig. 3b). There is no significant difference between 4TLM and 67NR groups in terms of E2F1 expression (Fig. 3b). All immunohistochemical

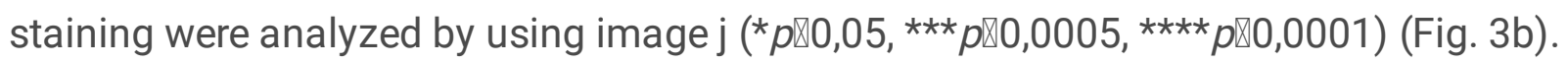

According to our results of immunohistochemistry in liver; FoxOs over expressions were observed especially in the infiltrated and metastatic cells existing the vessels (Fig. $3 \mathrm{C}$ ). While the expressions of Fox01, Fox03a and FoxO4 were observed in infiltrative cells around the hepatic artery, it was noteworthy that there was no expression in hepatocytes. There was no expression of cleaved caspase 3 between the

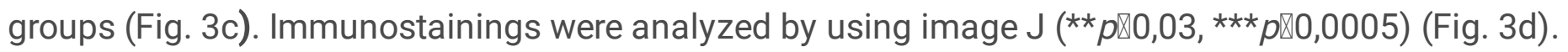

\subsubsection{Lung}

Expression of SIRT1 in lung tissue was extensively expressed in metastatic areas in the 4TLM metastatic cell line when compared to non-metastatic $67 \mathrm{NR}$ and tumor free groups. It was remarkable that SIRT1 expression was increased significantly in metastatic lesions in the 4TLM lung (Fig. 4a-4b) (**p $₫ 0,03$, $\star \star \star ~ p \llbracket 0,0005)$. p53 expression was observed in alveolar cells in the tumor free and 67NR groups. Although p53 expression was observed in the metastatic areas in the 4TLM group, it was lower compared to the tumor free group (Fig. 4a). There was no significant difference between groups in terms of p53 expression (Fig. 4b). Similar to p53 expression, p21 expression was higher in the tumor free group than in the 67NR and 4TLM group. In the 4TLM group, p21 was expressed more intensely in metastatic cells (Fig. 4a). It was determined that expression of p21 significantly decreased in 67NR group and 4TLM group compared to tumor free group (Fig. $4 \mathrm{~b})$ (** $p \llbracket 0,03, \star \star \star p \rrbracket 0,0005)$. Similarly, to SIRT1, E2F1 expression was observed intensely in metastatic areas in the 4TLM group. In tumor free and 67NR group, E2F1 expression was less (Fig. 4a). The difference of expressions between 4TLM and tumor free group was statistically significant (Fig. 4b) $\left({ }^{\star} p \bigotimes 0,05\right)$.

Fox01 expression was seen in metastatic cells in the 4TLM group. In addition, 67NR and tumor free group were negligible (Fig. 4c). Fox01 expressions were increased significantly in the 4TLM group compared to the tumor free group (Fig. 4d) ( $* \star p \otimes 0,03)$. Also, FoxO3 and FoxO4 were expressed in metastatic cells in the 4TLM group, similarly to Fox01. In the tumor free and 67NR group, their expressions were lower. The increase in FoxO3 expression in the 4TLM group was statistically significant compared to the tumor free group ( $\left.{ }^{\star \star \star} p \varangle 0,0005\right)$. In the tumor free group, there was a significant increase compared to the 67NR group (Fig. 4d) ( $\left.{ }^{\star} p \otimes 0,05\right)$. Fox04 expression was increased significantly in the

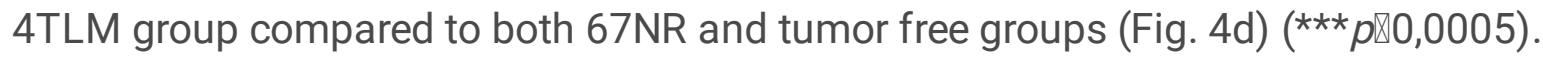

\subsection{Western blot findings: SIRT1, p53, p21, E2F1, and FoxOs expression levels in both 67NR and 4TLM primary tumors}

Expression levels of SIRT1, p53, p21, E2F1, and FoxO proteins were also analyzed western blotting from the primary tumors. The immunoblot signals for SIRT1, p53, p21, E2F1 and FoxO protein were decreased 
in the 67NR injected animal groups. GAPDH was used as internal control for all western blot analysis (Fig. 5). The blots revealed the bands for these proteins corresponding to $120 \mathrm{kDa}, 53 \mathrm{kDa}, 21 \mathrm{kDa}, 47$ $\mathrm{kDa}, 78-82 \mathrm{kDa}, 82-97 \mathrm{kDa}$, respectively. Equivalent amounts of total proteins were loaded per lane as indicative by the expression of GAPDH $(37 \mathrm{kDa})$. According to western blot results the expression patterns were confirmed to immunohistochemical staining's (Fig. 5a-b). SIRT1, p53, p21, E2F1 and FoxO1 and Fox03a are significantly decreased in 4TLM group compared to 67NR group ( ${ }^{\star \star} p \llbracket 0,03, \star \star \star p \llbracket 0,0005$, $\star \star \star \star p \otimes 0,0001)$.

\subsection{Real-time PCR findings: SIRT1, and FoxOs gene levels in both 67NR and 4TLM primary tumors}

The gene expression profiles of SIRT1, Fox01, FoxO3a, and FoxO4 gene in primary tumors were investigated real-time PCR analysis. The mRNA expression levels of SIRT1, Fox01 and Fox03a were significantly decreased in metastatic tumors compared to non-metastatic tumors; whereas FoxO4 signal was significantly increased in metastatic tumors (Fig. 6).

\subsection{Functional enrichment analysis findings}

To obtain an elementary investigation of the molecular mechanisms underlying Breast Cancer, microarray data were submitted to IPA core analysis. The differentially expressed genes were categorized to related canonical pathways based on Ingenuity pathway knowledge base. For DEGs mapped to IPA (genes not mapped to the IPA database were excluded in our pathway analysis), 113 significant canonical pathways were identified in three groups ( $\mathrm{BH}$ adjusted $\mathrm{p}$-value $<0.01$ ). The top enriched categories of canonical pathways with the absolute p-value and z-score more than 1.3 and 2, respectively, in primary breast cancer, liver and lung metastasis were presented by comparison analysis in Fig. 7. Calcium signaling was significantly increased in metastasis while Th1 signaling was significantly decreased compare to primary breast cancer. Hepatic fibrosis signaling and inflammation signaling has the higher activated score in metastatic liver tissue. Interestingly, Sirtuin Signaling Pathway, which modulates both the primary tumor and metastasis by stimulating or inhibiting the genes of FoxO and SIRT families, revealed the activated in three groups compatible with our results (Fig. 7, 8).

The upstream regulator analysis is a novel function in IPA by analyzing linkage to DEGs through coordinated expression; identify potential upstream regulators including transcription factors and genes that have been observed experimentally to affect gene expression. It has recently been used to robustly identify FoxO family as an important regulator in breast cancer and metastasis [45]. Fox01 ( $p$ value $=$ 1.19E-04, 5.16E-07), FoxO3 ( $p$ value $=2.94 \mathrm{E}-05,3.07 \mathrm{E}-07$ ), FoxO4 ( $\mathrm{p}$ value $=2.39 \mathrm{E}-08,1.0 \mathrm{E}-06$ ) were predicted upstream regulators in liver and lung metastasis, respectively, while only FoxO1 ( $p$ value $=2.6 \mathrm{E}-$ 03 ) and FoxO4 ( $p$ value $=4.59 \mathrm{E}-02$ ) were upstream regulators in primary breast tumor. Also, Sirt1 ( $p$ value $=4.46 \mathrm{E}-05)$ in primary tumor, SIRT1 and SIRT6 ( $p$ value $=3.4 \mathrm{E}-04,1.8 \mathrm{E}-01)$ in liver metastasis, and SIRT1 and SIRT 2 ( $p$ value $=3.27 \mathrm{E}-09,5.17 \mathrm{E}-03$ ) in lung metastasis played roles as upstream regulators. The target genes affected by Fox01, Fox03, FoxO4 and SIRT1 in liver and lung metastasis were shown in 
table 2. Regulator Effects with IPA explains how predicted activated or inhibited upstream regulators might cause increases or decreases in phenotypic or functional outcomes downstream. Cellular proliferation of tumor cells was shown as a regulator effect in metastases of 4T1 cell (Fig. 9).

\subsection{Clinic-pathological statistics of TNBC patients' findings}

Analysis of the mRNA expression of FoxO family and SIRT1 in TCGA revealed that all genes had downregulation in tumors compared to normal tissues in TNBC $(p<0.05$ for FoxO1, FoxO4) (Fig. 10). However, Fox01, Fox03, and SIRT1 were upregulated in metastasis stage of TNBC although the number of patients was too low to evaluate $(p>0.05)$.

\section{Discussion}

Breast cancer is one of the most common types of cancer in women in the world and second reason of cause of the death [2]. SIRT1 is known to regulate oncogenic signals and play a role in forming the appropriate microenvironment for tumor cell survival [46]. FoxO transcription factors are deacetylated by SIRT1 [15]. Deacetylated FoxO transcription factors also regulate cellular signals such as apoptosis, DNA damage, and cell survival $[10,16,17]$. The role of SIRT1 and FoxOs as regulators in important signaling pathways in tumor progression suggests that they are closely related to cancer. However, the effects of SIRT and FoxOs on metastasis in breast cancer have not been determined yet. Our purpose in this study is to explain the possible effects of SIRT1 and FoxOs on the progression and metastasis of breast cancer. In this study, firstly, we aimed to clarify the bidirectional role of SIRT1, which supports both tumor suppressor and tumor growth. Secondly, we aimed to understand the role of FoxO proteins during tumor progression and metastasis.

It is known that the increased activity of SIRT1 inhibits p53 by deacetylated. On the other hand, when SIRT1 activates p53, cancer formation is prevented by this activation $[46,47]$. Our results showed that both malignant and benign tumor cells express these molecules. SIRT1 expression was limited in the nucleus in 67NR cell lines. It is known that nuclear translocation is prevented by SIRT1 deacetylation, however it increases p53 accumulation in the cytoplasm [47]. In our study, keeping p53 in the cytoplasm while SIRT1 is in nucleus is related to these results. Similarly, it is known that p21 arrest the cell cycle when they are located in the cytoplasm [48]. In tumor cells, p21 and p53 are localized in the cytoplasm under in vitro conditions. In addition, more intensive expression of p21 and p53 in the cytoplasm of nonmetastatic tumor cells compared to metastatic tumor cells showed that 4TLM cells were more proliferative because the cell cycle continues rapidly in 4TLM cells.

Inhibition of SIRT1 is known to increase apoptosis by causing p53 activation. Also tumor growth reduces with this inhibition [49]. According to our results, SIRT1 expression was higher in 67NR compared to 4TLM. These results revealed that tumor suppressor activity of SIRT1 is active in non-metastatic groups. On the other hand, low expression of p53 and p21 may indicate that 4TLM tumor cells could escape from apoptosis. 
E2F1 is a key regulator of cell cycle [30]. Mori K. et al. showed to reduce cell proliferation by inhibition of E2F1 which is a key regulator of cell cycle in breast cancer cells [50]. According to our results, perinuclear localization of E2F1 in 4TLM primary tumors may reduce cell cycle. Our results regarding to p53, p21 and E2F1 indicated that metastatic 4TLM cells escape from apoptosis and continued their tumor progression. In contrast, cleaved caspase 3 expression was high in 4TLM primary tumors particularly in necrotic areas.

SIRT1 is known to be associated with metastasis, as well as their role in cell proliferation and tumor development. Jin X. et al. have been shown to trigger metastasis in breast cancer when SIRT1 expression is increased by lentivirus. High expression levels of SIRT1 causes increased invasion in breast cancer cells, while SIRT1 inhibition by shRNA decreases invasion of breast cancer cells. This evidence suggest that SIRT1 is associated with metastasis [51]. According to our results, the presence of SIRT1 expression in groups injected with 4TLM cells in the liver and lung is particularly noticeable. SIRT1 expression is very low in the 67NR groups and in tumor free groups. We also obtained that the level of SIRT1 expression was increased in metastasis of TNBC patients (Fig. 10B), while it was downregulated in primer tumor of TNBC patients compare to normal tissue (Fig. 10A). These results suggest that SIRT1 could be associated with metastasis, besides other roles.

FoxO transcription factors are known to function as tumor suppressors in cancers [45]. Localization of the FoxOs determine their activity status. FoxOs must be in the nucleus to activate its target genes which are related to crucial cellular processes. When FoxOs are translocated from nucleus to cytoplasm by growth factors, they cannot have function, resulting in inhibition of tumor suppressor function [52]. These results suggest that tumor suppressor FoxO proteins are retained in the cytoplasm and tumor cells are preserved.

Several studies shown to play a supportive role in facilitating and even stimulating metastasis for FoxOs [53-55]. While increased FoxO3a reduces metastasis, it is not affected to primary tumor growth [53]. In our study, Fox03a expressions were increased in non-metastatic 67NR compare to 4TLM primary tumors (Fig. 2D). In addition, FoxO1 and FoxO4 expressions in metastatic 4TLM primary tumors were lower than non-metastatic primary tumors. Similarly, the level of FoxO3 expression was decreased in metastasis of TNBC patients while detecting an increase in FoxO1, and FoxO4 expressions (Fig. 10B). Limited expression of FoxOs in metastatic tumors is thought to be closely related to the metastatic character of the tumor. Moreover, our results, also show that FoxO expressions are significantly higher in the 4TLM group compared to the non-metastatic and control groups in the liver which is one of the metastatic organs. The expressions of Fox01, FoxO3a and FoxO4 proteins were higher in the 4TLM lung especially in the metastatic cells. We obtained the same results in both liver and lung metastases of mice by transcriptomic analysis (Fig. 8, 9).

According to western blot results in primary tumors SIRT1, p21, p53, E2F1 and FoxO proteins were also higher in the 67NR group in primary tumors, similar to our immunohistochemistry results. Increased expressions of SIRT1, Fox01 and Fox03a in 67NR group supports our other results which are related to the metastatic character of the tumor. 
It has been shown that SIRT1 gene expression is high in 67NR group and may associated with the tumor suppressor effect of SIRT1 [49]. In our study, similar results were supported by immunohistochemistry, western blot and PCR analysis. Guttila I.K. et al., showed that Fox01 gene expression was higher in normal breast tissue than in breast tumor tissue [56]. In our results, FoxO1 expression increased in 67NR compared to 4TLM. Liu H. et al., showed that lower expression of FoxO3a promotes tumor progression by supporting stem cell characters of tumor cells. FoxO3a expressions down regulated in metastatic breast cancer tissues compared to normal breast epithelial cells (MCF-10A). Also, we observed that FoxO genes were downregulated in TNBC patients (Fig. 10A). In addition, with the downregulation of FoxO3 leads to change the levels of CD44 / CD24, which are breast cancer stem cell markers, was changed [57]. In our study, we showed FoxO3a expression was low in primary tumors obtained from 4TLM groups, which is highly metastatic. This result supports that FoxO3a affects the metastatic character of the tumor. In prostate cancer, FoxO4 mRNA expressions were increased in high grade tumor samples compared to benign prostate cancer [58]. In our results, observed that FoxO4 mRNA levels increased in the metastatic 4TLM group compared to the non-metastatic 67NR group. However, FoxO4 protein level decreased in 4TLM metastatic primary tumors.

The analysis of transcriptomic data revealed that some pathways are shared among primer tumor, metastatic lung and livers, but differences in transcript abundance existed (Fig. 7). The calcium signaling had a more enrichment score in metastases, while Th1 signal has more increased activation in primary tumor. It is known the potential role of T cells in promoting one of the most important steps in metastasis [59]. Th1 cells dramatically decrease the incidence of metastases while not altering the development of primary tumors [60]. Also, the contribution of the calcium signal to metastasis of cancer cells has been reviewed in previous studies [61]. Although physiological levels of $\mathrm{Ca} 2+$ inhibit proliferation and invasion, high $\mathrm{Ca} 2+$ levels ultimately increase the risk of metastasis in breast cancer [62]. Moreover, hepatic fibrosis signals, inflammation signals, and adipose tissue pathway were markedly more activated in metastatic liver tissue than others. By canonical pathway analysis, we obtained that Sirtuin signaling pathway, which consists of SIRT family members and FoxOs, was activated in both lung and liver metastasis (Fig. 8). Sirtuin pathway has a key role in regulation of genes involved in the metastatic processes in different cancers including breast cancer [63]. This path showed how cell proliferation and tumor growth are induced in lung and liver metastases coherent with our results (Fig. 9).

Furthermore, upstream regulator analysis by IPA identifies SIRT1, Fox01, Fox03, and FoxO4 as upstream regulators in lung and liver metastasis (Table 2). Yadav R. K. et al. reported that FoxOs generally serve as a central regulator of cellular homeostasis, cancer metabolism and are tumor suppressors in many of human primer cancers [45]. However, we detected that FoxOs genes regulated different many genes involved in the invasion, cell cycle, proliferation, apoptosis, ROS production, inflammation, and adipogenesis in metastases, concurrence with the experimental data in this study.

In conclusion, our results show that expressions of FoxO proteins were markedly lower in 4TLM groups compared to 67NR group. Decreased expression of FoxO proteins suggested that they could induced potential of metastasis. In the literature, it is emphasized that SIRT1 acts with FoxO4 and has a pro- 
apoptotic role and suppresses protease caspase 3 and 7 only in cancerous cells. The expression seen in metastatic liver tissues suggests that SIRT1 can deacetylate FoxO proteins by forming a complex in the nucleus with FoxO [10]. The expressions of SIRT1 and FoxO proteins in the lung and liver, especially metastatic cells, indicates that they may have crucial roles in metastasis [51, 53]. In addition, we show that FoxO1, FoxO3, FoxO4 and SIRT1 are associated with primary tumor and metastasis by IPA analysis and TCGA data. Our results, for the first time in the literature, shows that SIRT1 and FoxO proteins are associated with metastasis of breast cancer. Moreover, our findings will lead to further studies related to signaling mechanisms which still remains to investigate whether SIRT1 and FoxO proteins support metastasis.

\section{Abbreviations}

4TLM

4T1-Liver Metastasis Cell Line

4THM

4T1-Heart Metastasis Cell Line

67NR

Non-Metastatic Breast Cancer Cell Line

$\mathrm{BC}$

Breast Cancer

CDK1

Cyclin-dependent kinases 1 (p21)

$\mathrm{Ct}$

Cycle Threshold

$\mathrm{DAB}$

3,3'-Diaminobenzidine

DAPI

4',6-diamidino-2-phenylindole,

DEGs

Differentially Expressed Genes

DNA

Deoxyribonucleic acid

DMEM/F-12

Dulbecco's Modified Eagle Medium/Nutrient Mixture F-12

E2F

E2F Transcription Factor

E2F1

E2F Transcription Factor 1

FBS

Fetal Bovine Serum 
Fgf13

Fibroblast growth factor 13

FoxO

Forkhead box protein 0

Fox01

Forkhead box protein 0-1

FoxO3a

Forkhead box protein 0-3a

FoxO4

Forkhead box protein 0-4

$\mathrm{H}_{2} \mathrm{O}_{2}$

Hydrogen peroxide

HBSS

Hanks' Balanced Salt Solution

$\mathrm{HCl}$

Hydrochloric acid

HDAC

Histone Deacetylases

HRP

Horseradish peroxidase

$\lg \mathrm{G}$

Immunoglobulin G

IPA

Ingenuity Pathway Analysis

NAD

Nicotinamide adenine dinucleotide

OS

Overall survival

PBS

Phosphate-buffered saline

PVDF

Polyvinylidene fluoride

$\mathrm{Rb}$

Retinoblastoma

RNA

Ribonucleic acid

RT-PCR

Real Time Polymerase Chain Reaction

SDS

Sodium dodecyl sulfate 
SIRT1

Silent information regulator 1

TBS

Tris buffered saline

TRIS

Tris(hydroxymethyl)aminomethane

TSG

Tumor suppressor genes

\section{Declarations}

\section{ACKNOWLEDGEMENT}

This project is supported by Akdeniz University, The Scientific Research Projects Coordination Unit (Project number TSA-2017-2088).

\section{References}

1. Deshmukh SK, Srivastava SK, Poosarla T, Dyess DL, Holliday NP, Singh AP, Singh S. Inflammation, immunosuppressive microenvironment and breast cancer: opportunities for cancer prevention and therapy. Ann Transl Med. 2019;7(20):593.

2. Bray F, Ferlay J, Soerjomataram I, Siegel RL, Torre LA, Jemal A. Global cancer statistics 2018 : GLOBOCAN estimates of incidence and mortality worldwide for 36 cancers in 185 countries. CA Cancer J Clin. 2018;68(6):394-424.

3. Tan GZ, Li M, Tan X, Shi ML, Mou K. MiR-491 suppresses migration and invasion via directly targeting TPX2 in breast cancer. Eur Rev Med Pharmacol Sci. 2019;23(22):9996-10004.

4. Lee EY, Muller WJ. Oncogenes and tumor suppressor genes. Cold Spring Harb Perspect Biol. 2010;2(10):a003236.

5. Osborne C, Wilson P, Tripathy D. Oncogenes and tumor suppressor genes in breast cancer: potential diagnostic and therapeutic applications. Oncologist. 2004;9(4):361-77.

6. Blander G, Guarente L. The Sir2 family of protein deacetylases. Annu Rev Biochem. 2004;73:417-35.

7. Cho IR, Koh SS, Malilas W, Srisuttee R, Moon J, Choi YW, Horio Y, Oh S, Chung YH. SIRT1 inhibits proliferation of pancreatic cancer cells expressing pancreatic adenocarcinoma up-regulated factor (PAUF), a novel oncogene, by suppression of beta-catenin. Biochem Biophys Res Commun. 2012;423(2):270-5.

8. Luo J, Su F, Chen D, Shiloh A, Gu W. Deacetylation of p53 modulates its effect on cell growth and apoptosis. Nature. 2000;408(6810):377-81.

9. Peck B, Chen CY, Ho KK, Di Fruscia P, Myatt SS, Coombes RC, Fuchter MJ, Hsiao CD, Lam EW. SIRT inhibitors induce cell death and p53 acetylation through targeting both SIRT1 and SIRT2. Mol Cancer 
Ther. 2010;9(4):844-55.

10. Brunet A, Sweeney LB, Sturgill JF, Chua KF, Greer PL, Lin Y, Tran H, Ross SE, Mostoslavsky R, Cohen HY, et al. Stress-dependent regulation of FOXO transcription factors by the SIRT1 deacetylase. Science. 2004;303(5666):2011-5.

11. Motta MC, Divecha N, Lemieux M, Kamel C, Chen D, Gu W, Bultsma Y, McBurney M, Guarente L. Mammalian SIRT1 represses forkhead transcription factors. Cell. 2004;116(4):551-63.

12. Carter ME, Brunet A. FOXO transcription factors. Curr Biol. 2007;17(4):R113-4.

13. Medema RH, Jaattela M. Cytosolic Fox01: alive and killing. Nat Cell Biol. 2010;12(7):642-3.

14. Barthel A, Schmoll D, Unterman TG. FoxO proteins in insulin action and metabolism. Trends Endocrinol Metab. 2005;16(4):183-9.

15. Kobayashi Y, Furukawa-Hibi Y, Chen C, Horio Y, Isobe K, Ikeda K, Motoyama N. SIRT1 is critical regulator of FOXO-mediated transcription in response to oxidative stress. Int $\mathrm{J}$ Mol Med. 2005;16(2):237-43.

16. Miyamoto K, Araki KY, Naka K, Arai F, Takubo K, Yamazaki S, Matsuoka S, Miyamoto T, Ito K, Ohmura $\mathrm{M}$, et al. Foxo3a is essential for maintenance of the hematopoietic stem cell pool. Cell Stem Cell. 2007;1(1):101-12.

17. Ford J, Jiang M, Milner J. Cancer-specific functions of SIRT1 enable human epithelial cancer cell growth and survival. Cancer Res. 2005;65(22):10457-63.

18. Choi HK, Cho KB, Phuong NT, Han CY, Han HK, Hien TT, Choi HS, Kang KW. SIRT1-mediated FoxO1 deacetylation is essential for multidrug resistance-associated protein 2 expression in tamoxifenresistant breast cancer cells. Mol Pharm. 2013;10(7):2517-27.

19. Hu Q, Wang G, Peng J, Qian G, Jiang W, Xie C, Xiao Y, Wang X. Knockdown of SIRT1 Suppresses Bladder Cancer Cell Proliferation and Migration and Induces Cell Cycle Arrest and Antioxidant Response through FOX03a-Mediated Pathways. Biomed Res Int. 2017;2017:3781904.

20. Zhao G, Cui J, Zhang JG, Qin Q, Chen Q, Yin T, Deng SC, Liu Y, Liu L, Wang B, et al. SIRT1 RNAi knockdown induces apoptosis and senescence, inhibits invasion and enhances chemosensitivity in pancreatic cancer cells. Gene Ther. 2011;18(9):920-8.

21. Saretzki G. Cellular senescence in the development and treatment of cancer. Curr Pharm Des. 2010;16(1):79-100.

22. Gonfloni S, Iannizzotto V, Maiani E, Bellusci G, Ciccone S, Diederich M. P53 and Sirt1: routes of metabolism and genome stability. Biochem Pharmacol. 2014;92(1):149-56.

23. Sarma P, Bag I, Ramaiah MJ, Kamal A, Bhadra U, Pal Bhadra M. Bisindole-PBD regulates breast cancer cell proliferation via SIRT-p53 axis. Cancer Biol Ther. 2015;16(10):1486-501.

24. Li K, Luo J. The role of SIRT1 in tumorigenesis. N Am J Med Sci (Boston). 2011;4(2):104-6.

25. Abbas T, Dutta A. p21 in cancer: intricate networks and multiple activities. Nat Rev Cancer. 2009;9(6):400-14. 
26. Xiong Y, Zhang H, Beach D. D type cyclins associate with multiple protein kinases and the DNA replication and repair factor PCNA. Cell. 1992;71(3):505-14.

27. Di Agostino S, Strano S, Emiliozzi V, Zerbini V, Mottolese M, Sacchi A, Blandino G, Piaggio G. Gain of function of mutant p53: the mutant $\mathrm{p} 53 / \mathrm{NF}-\mathrm{Y}$ protein complex reveals an aberrant transcriptional mechanism of cell cycle regulation. Cancer Cell. 2006;10(3):191-202.

28. Bourgeois B, Madl T. Regulation of cellular senescence via the FOXO4-p53 axis. FEBS Lett. 2018;592(12):2083-97.

29. Biswas AK, Johnson DG. Transcriptional and nontranscriptional functions of E2F1 in response to DNA damage. Cancer Res. 2012;72(1):13-7.

30. Ertosun MG, Hapil FZ, Osman Nidai O. E2F1 transcription factor and its impact on growth factor and cytokine signaling. Cytokine Growth Factor Rev. 2016;31:17-25.

31. Hazar-Rethinam M, Endo-Munoz L, Gannon O, Saunders N. The role of the E2F transcription factor family in UV-induced apoptosis. Int J Mol Sci. 2011;12(12):8947-60.

32. Chen D, Padiernos E, Ding F, Lossos IS, Lopez CD. Apoptosis-stimulating protein of p53-2 (ASPP2/53BP2L) is an E2F target gene. Cell Death Differ. 2005;12(4):358-68.

33. Fogal V, Kartasheva NN, Trigiante G, Llanos S, Yap D, Vousden KH, Lu X. ASPP1 and ASPP2 are new transcriptional targets of E2F. Cell Death Differ. 2005;12(4):369-76.

34. Lee JS, Leem SH, Lee SY, Kim SC, Park ES, Kim SB, Kim SK, Kim YJ, Kim WJ, Chu IS. Expression signature of E2F1 and its associated genes predict superficial to invasive progression of bladder tumors. J Clin Oncol. 2010;28(16):2660-7.

35. Hallstrom TC, Mori S, Nevins JR. An E2F1-dependent gene expression program that determines the balance between proliferation and cell death. Cancer Cell. 2008;13(1):11-22.

36. Alla V, Engelmann D, Niemetz A, Pahnke J, Schmidt A, Kunz M, Emmrich S, Steder M, Koczan D, Putzer BM. E2F1 in melanoma progression and metastasis. J Natl Cancer Inst. 2010;102(2):127-33.

37. Hollern DP, Swiatnicki MR, Rennhack JP, Misek SA, Matson BC, McAuliff A, Gallo KA, Caron KM, Andrechek ER. E2F1 Drives Breast Cancer Metastasis by Regulating the Target Gene FGF13 and Altering Cell Migration. Sci Rep. 2019;9(1):10718.

38. Aslakson CJ, Miller FR. Selective events in the metastatic process defined by analysis of the sequential dissemination of subpopulations of a mouse mammary tumor. Cancer research. 1992;52(6):1399-405.

39. Erin N, Boyer PJ, Bonneau RH, Clawson GA, Welch DR. Capsaicin-mediated denervation of sensory neurons promotes mammary tumor metastasis to lung and heart. Anticancer research. 2004;24(2B):1003-9.

40. Erin N, Wang N, Xin P, Bui V, Weisz J, Barkan GA, Zhao W, Shearer D, Clawson GA. Altered gene expression in breast cancer liver metastases. International journal of cancer. 2009;124(7):1503-16.

41. Cuesta S, Kireev R, Garcia C, Rancan L, Vara E, Tresguerres JA: Melatonin can improve insulin resistance and aging-induced pancreas alterations in senescence-accelerated prone male mice 
(SAMP8). Age (Dordr) 2013, 35(3):659-671.

42. Zhu J, Jiang X, Chehab FF. FoxO4 interacts with the sterol regulatory factor SREBP2 and the hypoxia inducible factor HIF2alpha at the CYP51 promoter. Journal of lipid research. 2014;55(3):431-42.

43. Ritchie ME, Phipson B, Wu D, Hu Y, Law CW, Shi W, Smyth GK. limma powers differential expression analyses for RNA-sequencing and microarray studies. Nucleic Acids Res. 2015;43(7):e47.

44. Tang Z, Li C, Kang B, Gao G, Li C, Zhang Z. GEPIA: a web server for cancer and normal gene expression profiling and interactive analyses. Nucleic Acids Res. 2017;45(W1):W98-102.

45. Yadav RK, Chauhan AS, Zhuang L, Gan B. FoxO transcription factors in cancer metabolism. Semin Cancer Biol. 2018;50:65-76.

46. Wilking MJ, Singh C, Nihal M, Zhong W, Ahmad N. SIRT1 deacetylase is overexpressed in human melanoma and its small molecule inhibition imparts anti-proliferative response via p53 activation. Arch Biochem Biophys. 2014;563:94-100.

47. Yi J, Luo J. SIRT1 and p53, effect on cancer, senescence and beyond. Biochim Biophys Acta. 2010;1804(8):1684-9.

48. Green DR, Kroemer G. Cytoplasmic functions of the tumour suppressor p53. Nature. 2009;458(7242):1127-30.

49. Lin Z, Fang D. The Roles of SIRT1 in Cancer. Genes Cancer. 2013;4(3-4):97-104.

50. Mori K, Uchida T, Fukumura M, Tamiya S, Higurashi M, Sakai H, Ishikawa F, Shibanuma M. Linkage of E2F1 transcriptional network and cell proliferation with respiratory chain activity in breast cancer cells. Cancer Sci. 2016;107(7):963-71.

51. Jin X, Wei Y, Xu F, Zhao M, Dai K, Shen R, Yang S, Zhang N. SIRT1 promotes formation of breast cancer through modulating Akt activity. J Cancer. 2018;9(11):2012-23.

52. Farhan M, Wang H, Gaur U, Little PJ, Xu J, Zheng W. FOXO Signaling Pathways as Therapeutic Targets in Cancer. Int J Biol Sci. 2017;13(7):815-27.

53. Storz P, Doppler H, Copland JA, Simpson KJ, Toker A. FOXO3a promotes tumor cell invasion through the induction of matrix metalloproteinases. Mol Cell Biol. 2009;29(18):4906-17.

54. Hornsveld M, Dansen TB, Derksen PW, Burgering BMT. Re-evaluating the role of FOXOs in cancer. Semin Cancer Biol. 2018;50:90-100.

55. Feng X, Wu Z, Wu Y, Hankey W, Prior TW, Li L, Ganju RK, Shen R, Zou X. Cdc25A regulates matrix metalloprotease 1 through Foxo1 and mediates metastasis of breast cancer cells. Mol Cell Biol. 2011;31(16):3457-71.

56. Guttilla IK, White BA. Coordinate regulation of FOXO1 by miR-27a, miR-96, and miR-182 in breast cancer cells. J Biol Chem. 2009;284(35):23204-16.

57. Liu H, Song Y, Qiu H, Liu Y, Luo K, Yi Y, Jiang G, Lu M, Zhang Z, Yin J, et al. Downregulation of FOXO3a by DNMT1 promotes breast cancer stem cell properties and tumorigenesis. Cell Death Differ. 2020;27(3):966-83. 
58. Shukla S, Shukla M, Maclennan GT, Fu P, Gupta S. Deregulation of FOX03A during prostate cancer progression. Int J Oncol. 2009;34(6):1613-20.

59. Pardoll D. Metastasis-promoting immunity: when T cells turn to the dark side. Cancer Cell. 2009;16(2):81-2.

60. DeNardo DG, Barreto JB, Andreu P, Vasquez L, Tawfik D, Kolhatkar N, Coussens LM. CD4(+) T cells regulate pulmonary metastasis of mammary carcinomas by enhancing protumor properties of macrophages. Cancer Cell. 2009;16(2):91-102.

61. Bong AHL, Monteith GR. Calcium signaling and the therapeutic targeting of cancer cells. Biochim Biophys Acta Mol Cell Res. 2018;1865(11 Pt B):1786-94.

62. Journe F, Dumon JC, Kheddoumi N, Fox J, Laios I, Leclercq G, Body JJ. Extracellular calcium downregulates estrogen receptor alpha and increases its transcriptional activity through calciumsensing receptor in breast cancer cells. Bone. 2004;35(2):479-88.

63. Sinha S, Sharma S, Vora J, Shrivastava N. Emerging role of sirtuins in breast cancer metastasis and multidrug resistance: Implication for novel therapeutic strategies targeting sirtuins. Pharmacol Res. 2020;158:104880.

\section{Tables}

Due to technical limitations, table 1 and 2 is only available as a download in the Supplemental Files section.

\section{Figures}


a
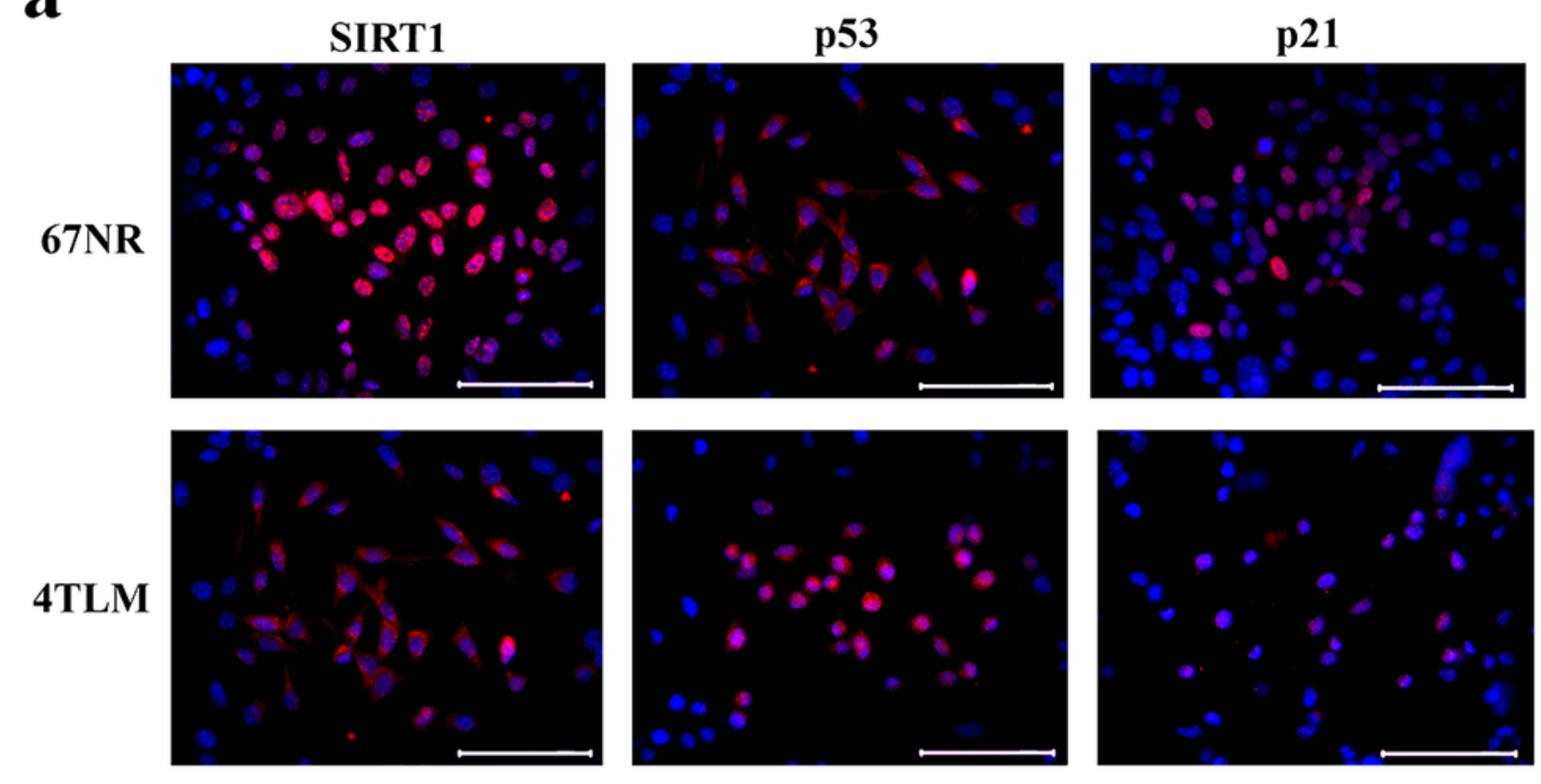

b
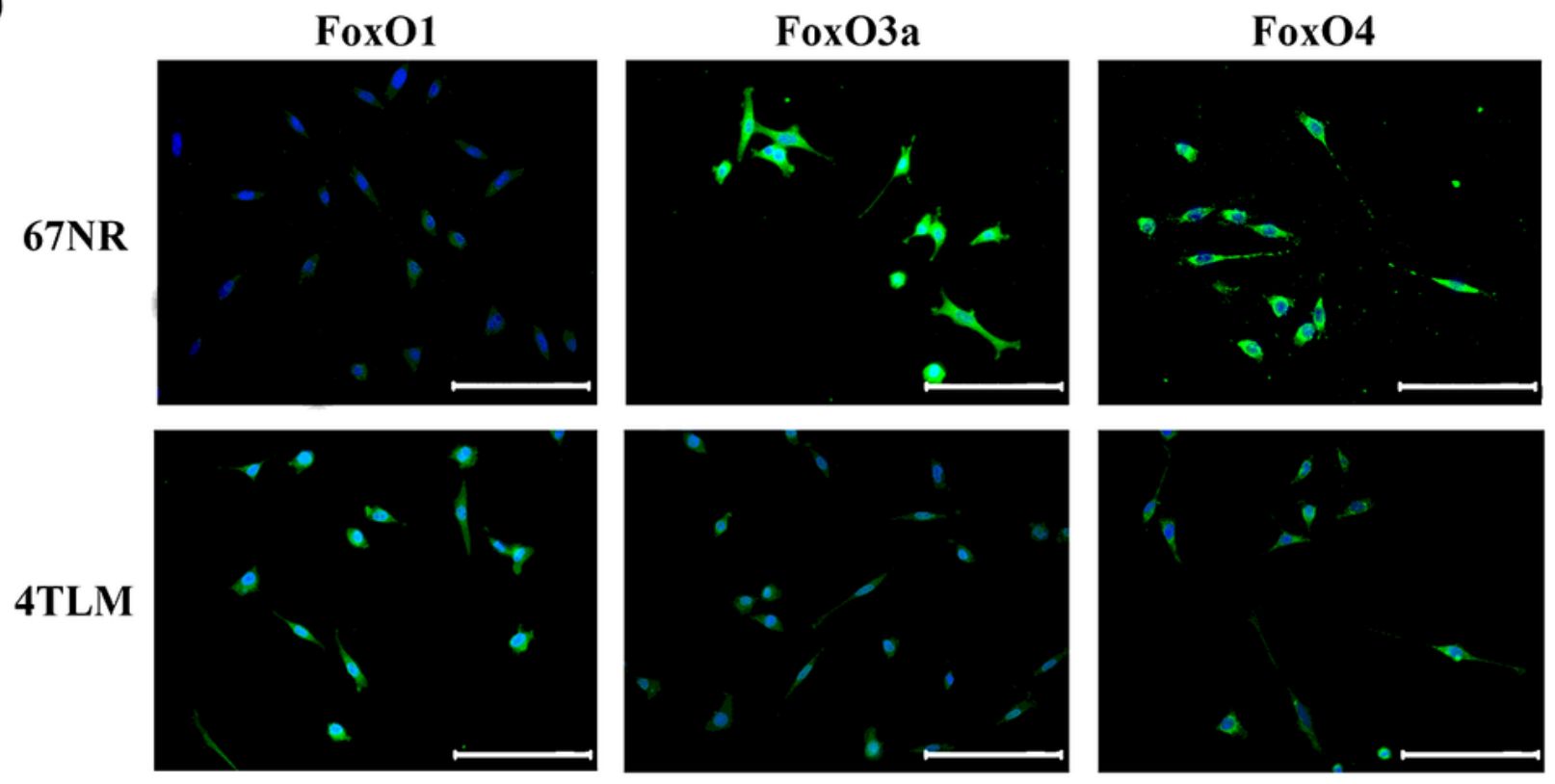

\section{Figure 1}

Immunofluorescence labelling of SIRT1, p53, p21 and FoxO proteins in 4TLM and 67NR cell lines. A) Representative images show in SIRT1, p53 and p21 expressions 67NR and 4TLM cancer cells. Red signal represents target protein expression and blue signal represents DAPI staining which was used to stain the

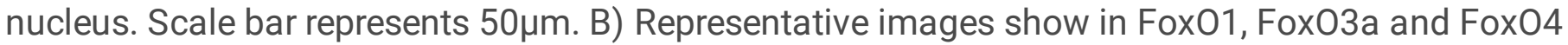


expressions of 67NR and 4TLM. Green signal represents target protein expression and blue signal represents DAPI staining which was used to stain the nucleus.
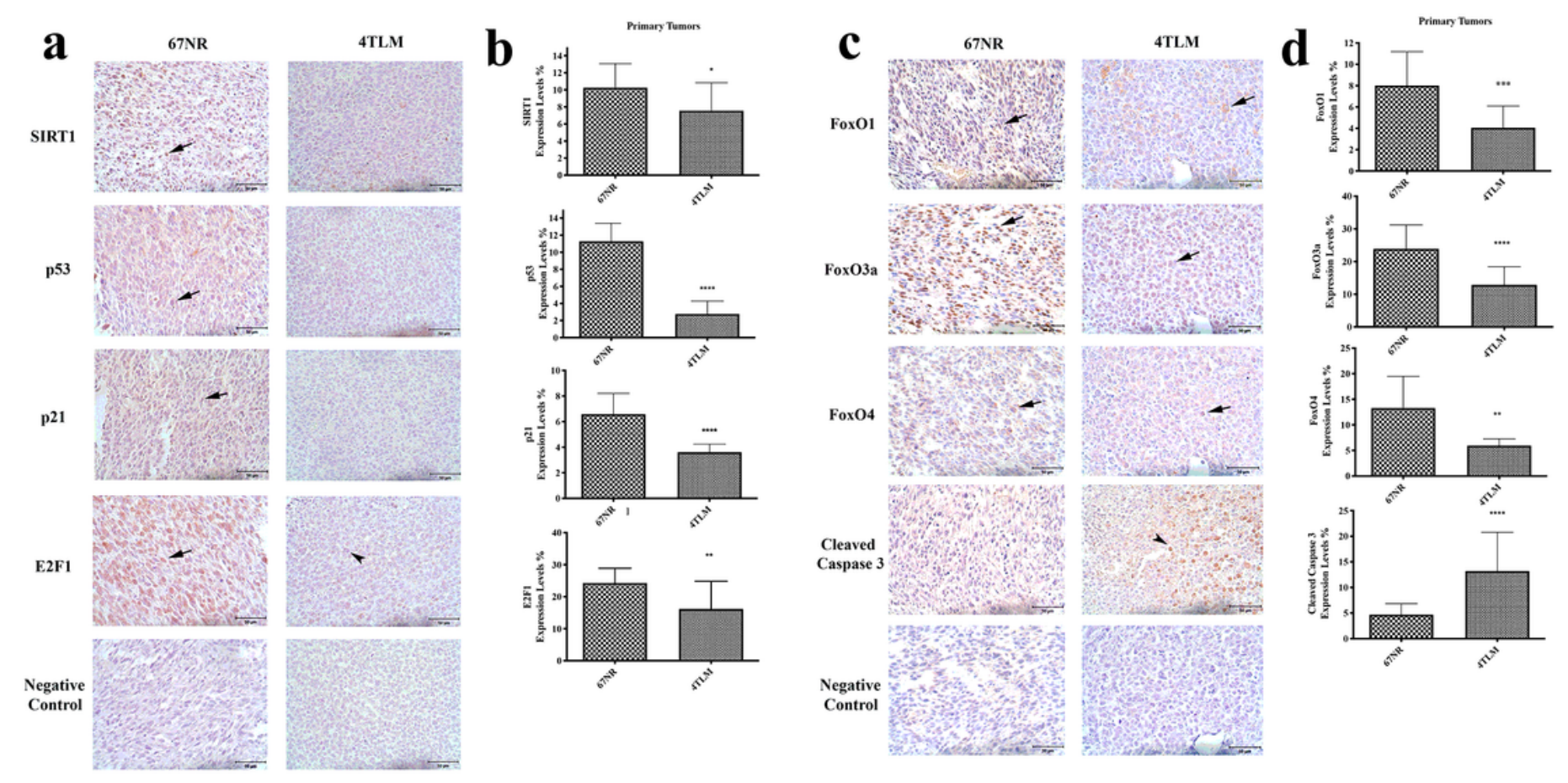

\section{Figure 2}

Expression of SIRT1 p53, p21, E2F1, FoxO proteins and cleaved caspase 3 in 67NR and 4TLM primary tumors. A) Representative images show in SIRT1, p53, p21 and E2F1 expressions in 67NR and 4TLM primary tumors. Arrow represent target protein expressions for each protein expressions and arrowhead represents perinuclear E2F1 expression in 4TLM B) Graphs demonstrate the results of image $\mathrm{J}$ analysis

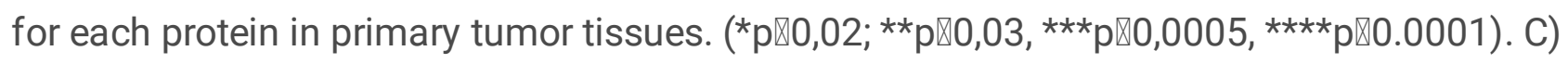
Representative images show in Fox01, FoxO3a, FoxO4 and cleaved caspase 3 proteins in 67NR and 4TLM primary tumors. Arrow represent target protein expressions for each protein expressions and arrowhead represents nuclear cleaved caspase3 expression in 4TLM. D) Graphs demonstrate results of image $\mathrm{J}$ analysis for each protein in primary tumors ( ${ }^{*} \mathrm{p} \otimes 0,03,{ }^{* \star *} \mathrm{p} \otimes 0,0005,{ }^{* \star \star *} \mathrm{p} \otimes 0.0001$ ). 

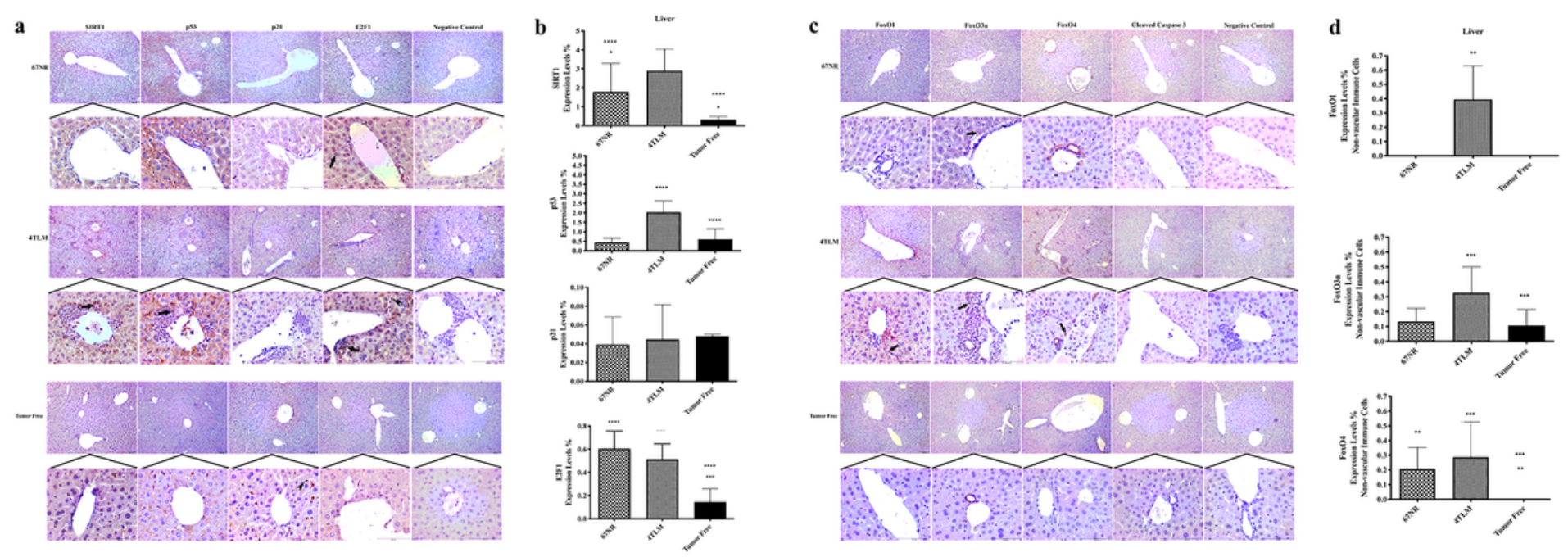

\section{Figure 3}

A) Representative figures of SIRT1, p53, p21 and E2F1 immunohistochemical staining of liver tissues. Immunohistochemical reactions have been interpreted compared to animals injected with both metastatic and non-metastatic cell lines in addition to tumor free animals. Arrow represent target protein expressions for each protein expressions in infiltrated cells, arrowhead represents Kupffer cells. B) Graphs demonstrate the results of image $J$ analysis for each protein in liver tissues $\left({ }^{\star} p \otimes 0,05,{ }^{\star} \star \star p \otimes 0,0005\right.$, $* \star \star \star p \otimes 0,0001)$. C) Representative figures of Fox01, Fox03a, FoxO4 and Cleaved caspase 3 proteins immunohistochemical staining of liver tissues. Arrow represent target protein expressions for each protein expressions in infiltrated cells. D) Graphs showed the results of image $\mathrm{J}$ analysis for each protein

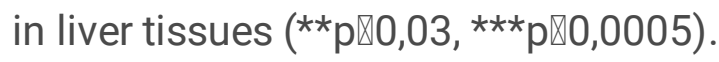
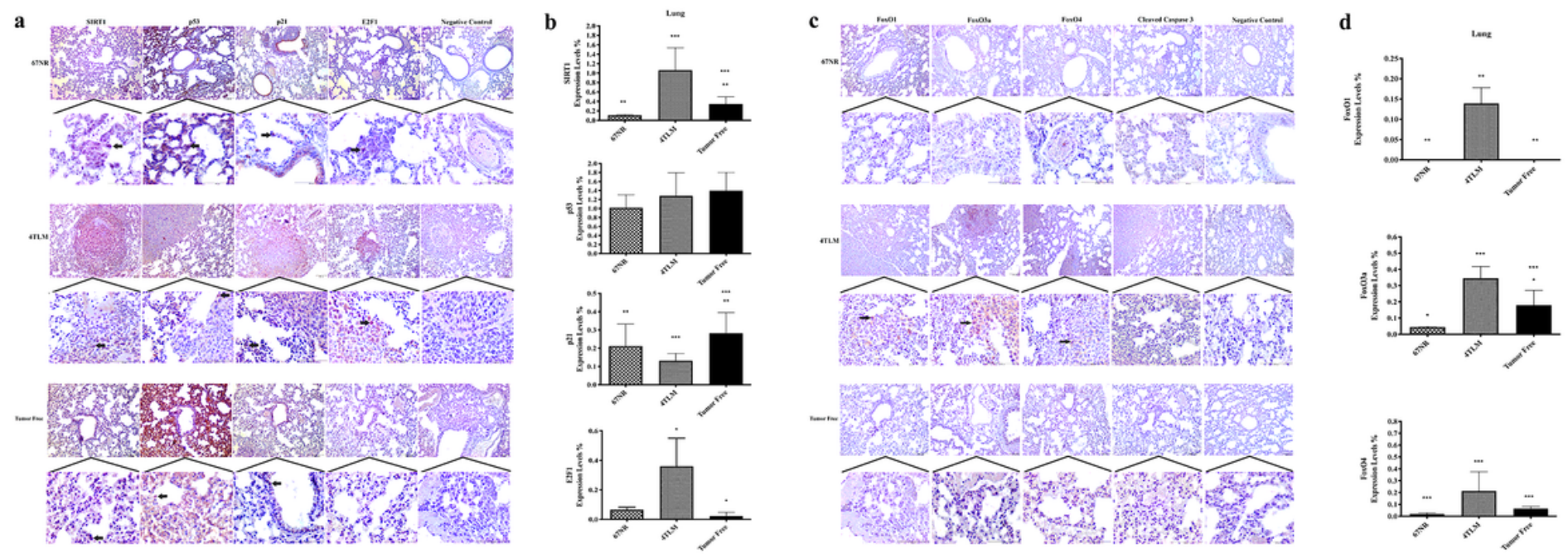

\section{Figure 4}

A) Representative figure of SIRT1, p53, p21 and E2F1 in the lung tissue. Arrow represent target protein expressions for each protein expressions in infiltrated cells. B) The graphs demonstrate the results of

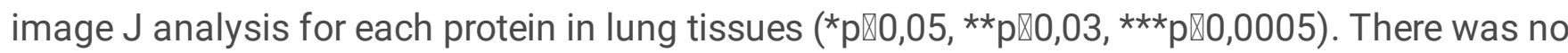


significantly difference in p53 expression. C) Representative figure of Fox01, Fox03a, FoxO4 and Cleaved caspase 3 in the lung tissue. Arrow represent target protein expressions for each protein expressions in infiltrated cells. D) Graphs demonstrate the immunohistochemical analysis results of image $\mathrm{J}$ analysis for each FoxO proteins in lung tissues of ( $\left.{ }^{*} \mathrm{p} \otimes 0,05,{ }^{* *} p \otimes 0,03,{ }^{* * *} \mathrm{p} \otimes 0,0005\right)$.

a

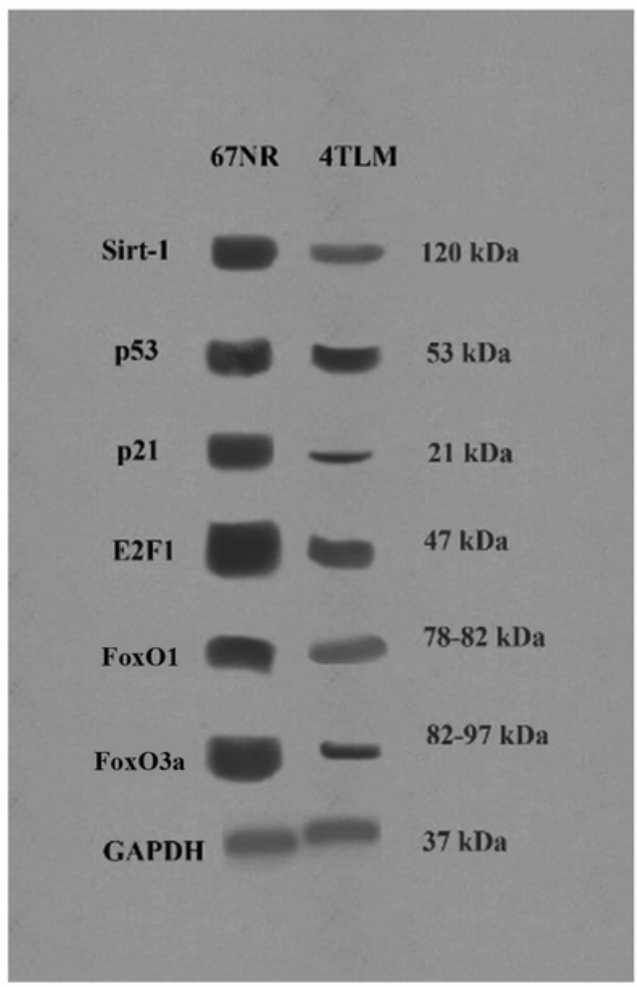

b

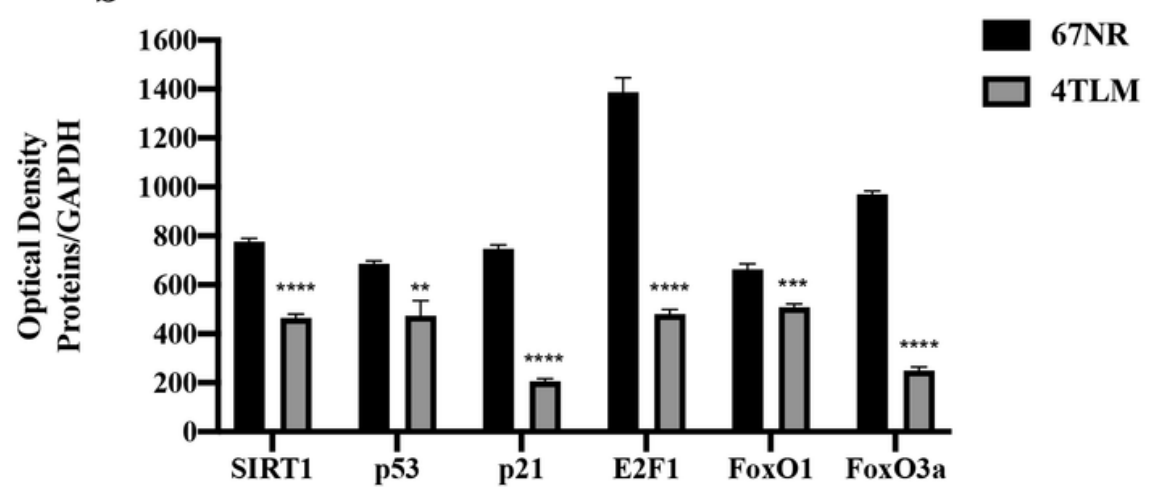

Figure 5

Western blot analysis of the SIRT1, p53, p21, E2F1, FoxO1 and FoxO3 proteins in 67NR and 4TLM primary tumors. A) The bands represent of SIRT1, p53, p21, E2F1, FoxO1 and FoxO3a proteins corresponding to $120 \mathrm{kDa}, 53 \mathrm{kDa}, 21 \mathrm{kDa}, 47 \mathrm{kDa}, 78-82 \mathrm{kDa}, 82-97 \mathrm{kDa}$, respectively. GAPDH (37kDa) was used as a loading control. B) SIRT1, p53, p21, E2F1 and FoxO1 and FoxO3a is decreased in 4TLM

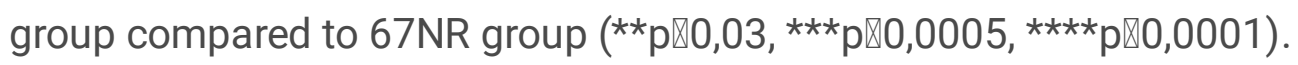




\section{TLM/67NR Fold Change $(\Delta \Delta \mathbf{C t})$}

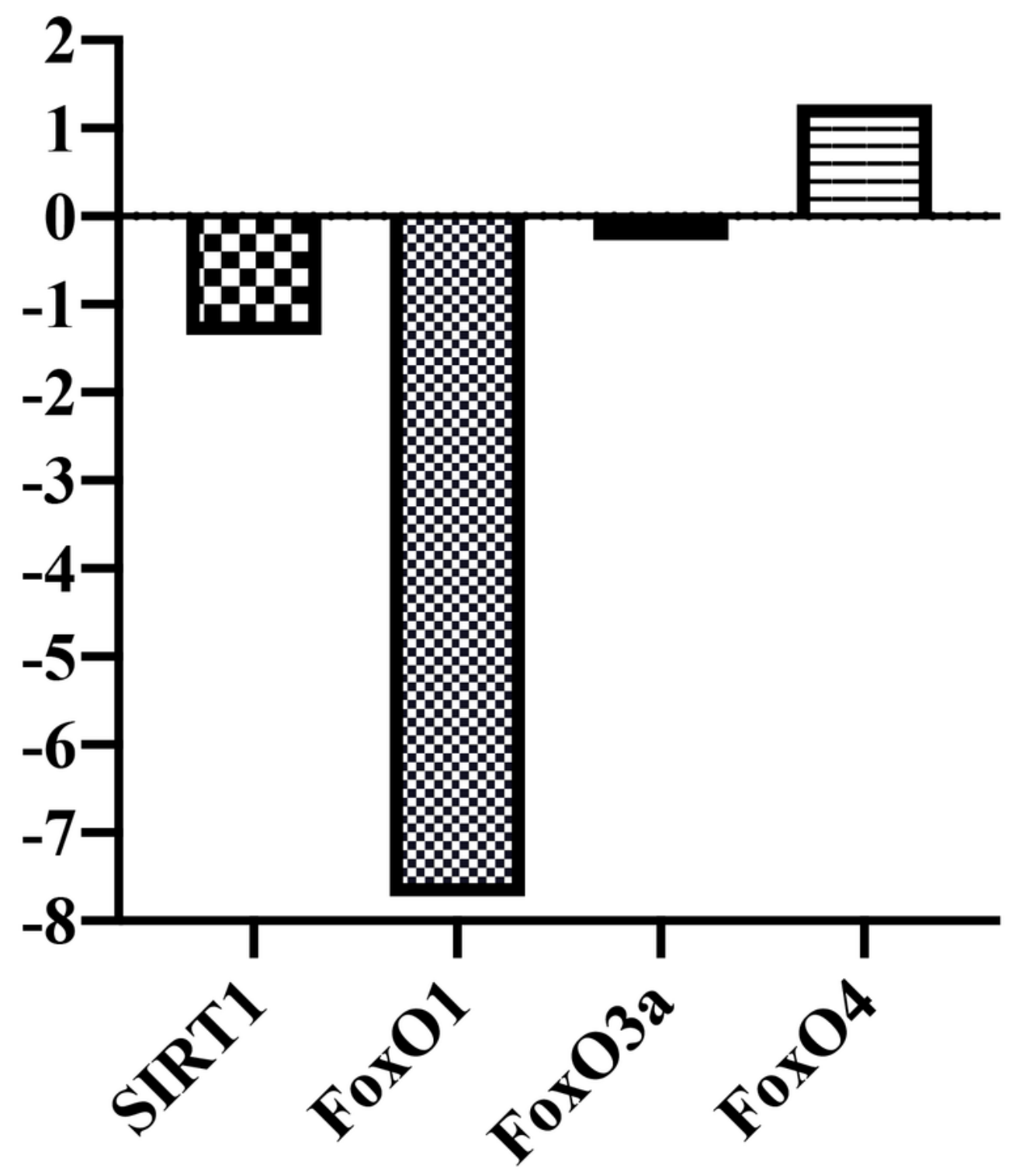

Figure 6

The graph represents mRNA levels of SIRT1, Fox01 and FoxO3a and FoxO4. 
a

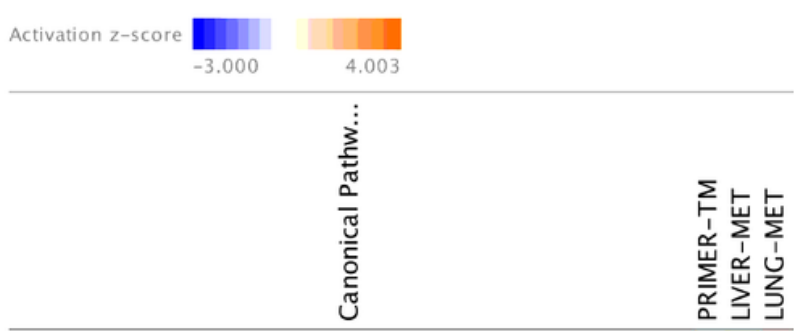

White Adipose Tissue Browning Pathway Thyroid Cancer Signaling

HMGB1 Signaling

eNOS Signaling

Retinoate Biosynthesis I

Neuroinflammation Signaling Pathway

Hepatic Fibrosis Signaling Pathway

Eicosanoid Signaling

FGF Signaling

Th1 Pathway

G Beta Gamma Signaling

Calcium Signaling

Bladder Cancer Signaling

Adrenomedullin signaling pathway

Estrogen Receptor Signaling

Melanocyte Development and Pigmentation Signaling

Colorectal Cancer Metastasis Signaling

CAMP-mediated signaling

Phospholipases

Signaling by Rho Family GTPases

Antioxidant Action of Vitamin C

FAT10 Cancer Signaling Pathway

TREM1 Signaling

Histamine Degradation

Opioid Signaling Pathway

Inflammasome pathway

Apelin Pancreas Signaling Pathway

Leukotriene Biosynthesis b

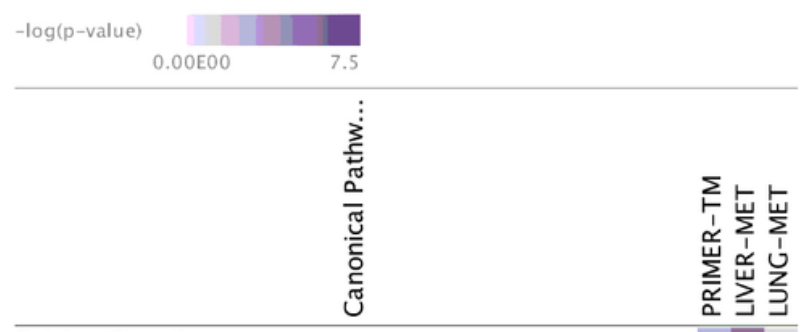

Calcium Signaling

White Adipose Tissue Browning Pathway

Neuroinflammation Signaling Pathway

CAMP-mediated signaling

Hepatic Fibrosis Signaling Pathway

Bladder Cancer Signaling

Th1 Pathway

Eicosanoid Signaling

TREM1 Signaling

eNOS Signaling

Colorectal Cancer Metastasis Signaling

Retinoate Biosynthesis I

Inflammasome pathway

HMGB1 Signaling

Histamine Degradation

Phospholipases

Adrenomedullin signaling pathway

Estrogen Receptor Signaling

G Beta Gamma Signaling

Opioid Signaling Pathway

FAT10 Cancer Signaling Pathway

Leukotriene Biosynthesis

Antioxidant Action of Vitamin C

Signaling by Rho Family GTPases

Thyroid Cancer Signaling

Apelin Pancreas Signaling Pathway

FGF Signaling

Melanocyte Development and Pigmentation Signaling

\section{Figure 7}

Canonical pathways comparison from 4T1 primary tumor and metastatic subpopulations by IPA in GSE62598 data. Heat maps showed A) activation score (absolute z-score $>2$ ) and B) p-value (absolute log p-value>1.3). 


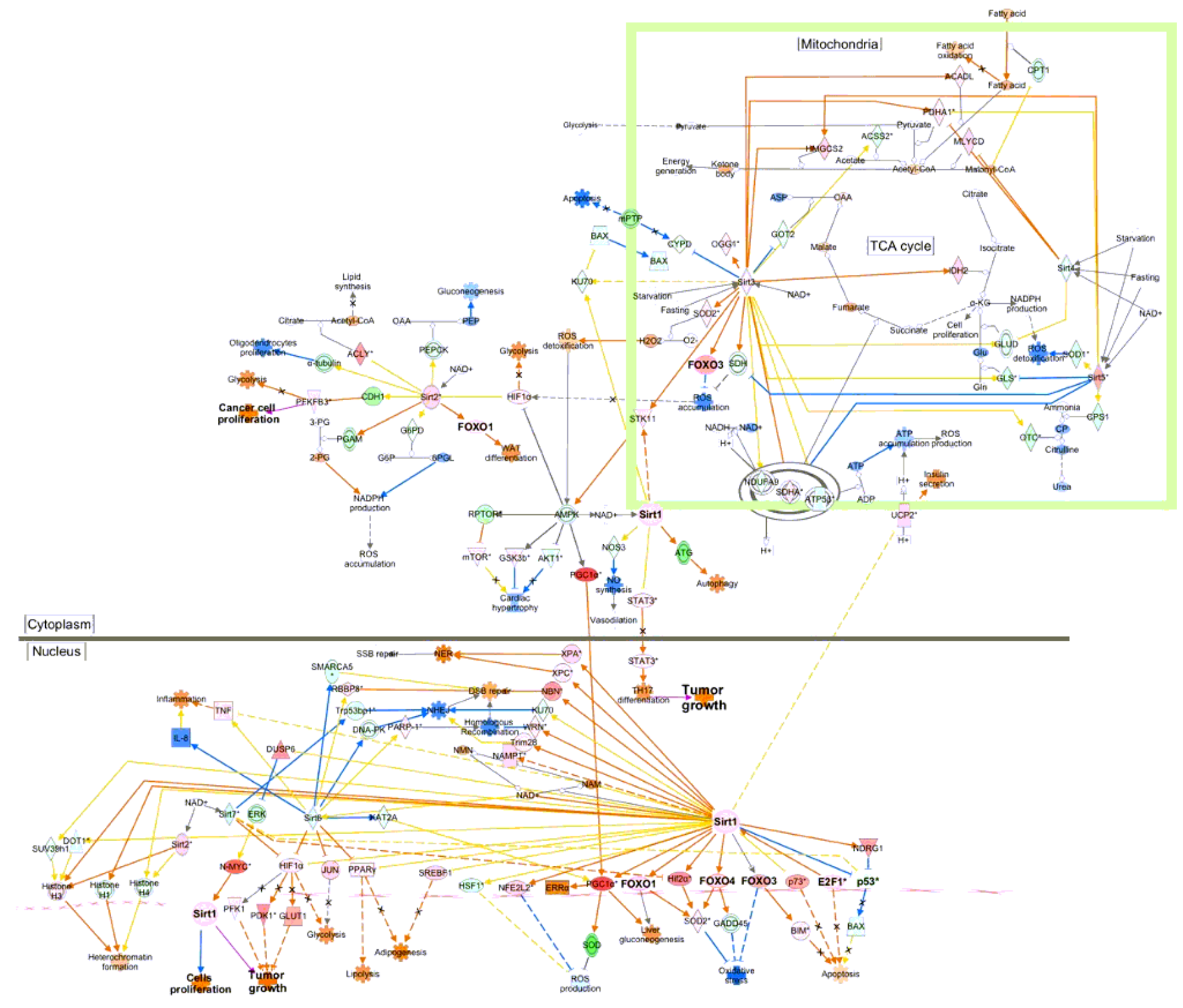

Figure 8

Sirtuin pathway was generated from GSE62598 data that included gene expression profile of 4T1 primary tumor and liver/lung metastatic sub-populations in mouse model. Figure represents gene profiling's in liver metastasis of $4 \mathrm{~T} 1$ cell (absolute log p-value $>1.3$ ). 
SIRT3 CDKN1A E2F1* FOXO4 SIRT6 CASP3* SIRT4 SIRT2* FOXO1, FOXO3, SIRT1 SIRT7* Liver_Metastasis SIRT3 CDKNTÁ E2F1 FOXO4 SIRT́ CASP'

\section{Cell proliferation of tumor cell}

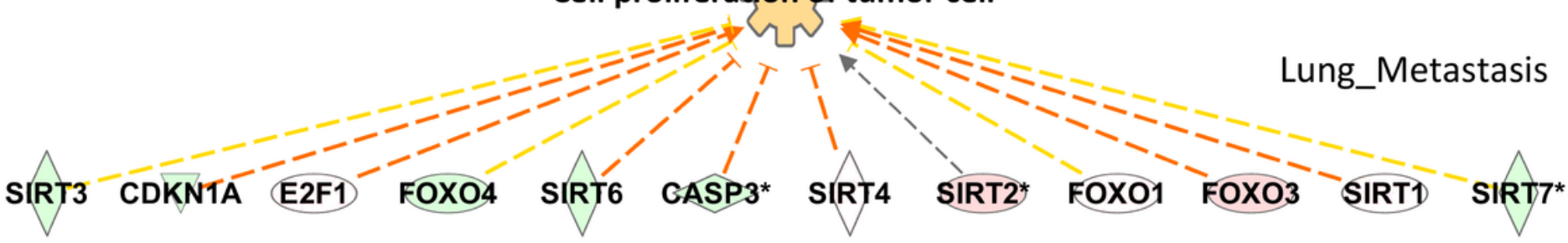

\section{Figure 9}

Cell proliferation of tumor cells was detected as a Regulator Effects in liver and lung metastases of 4T1 cell by IPA.

a
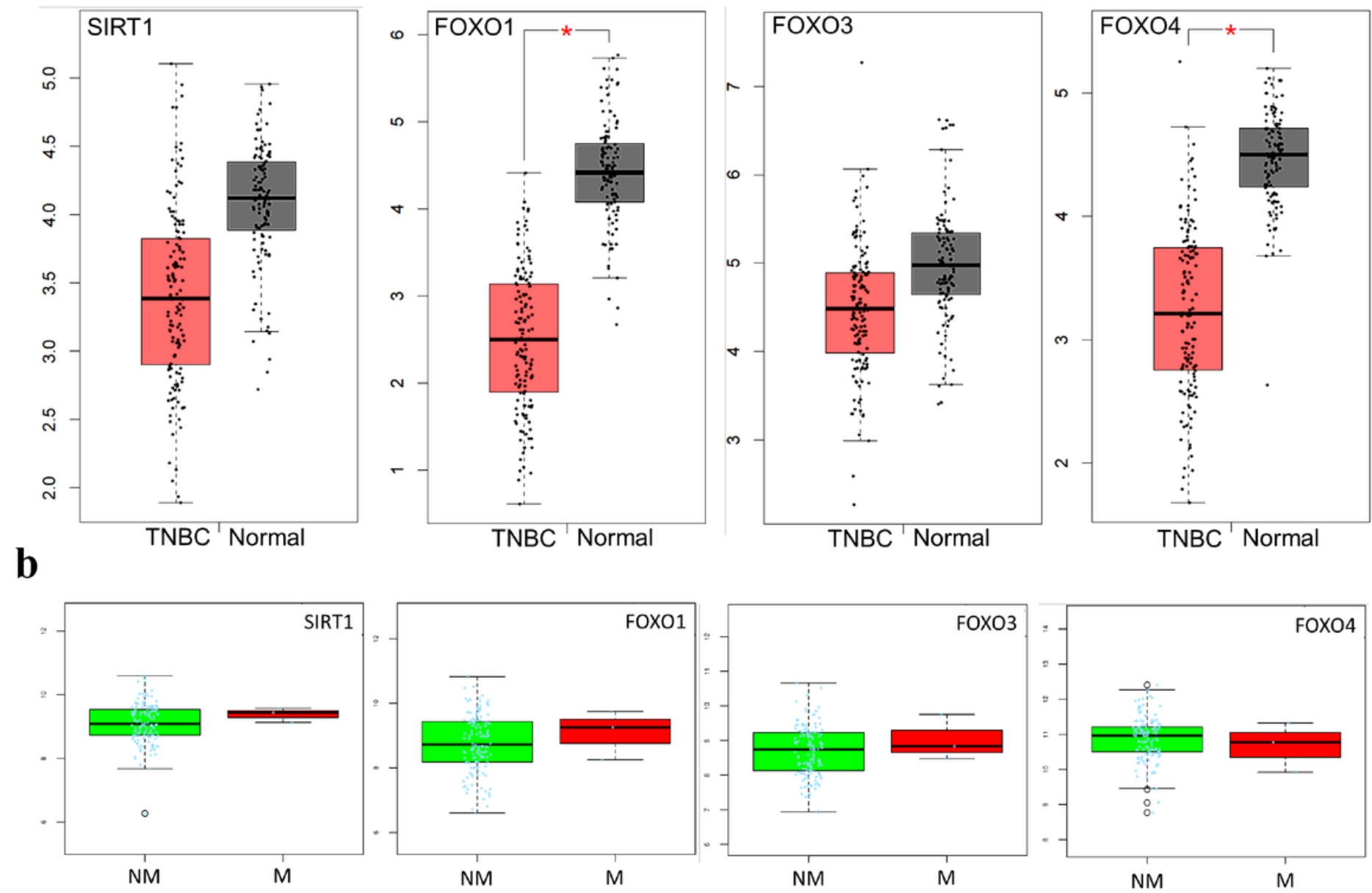

Figure 10 
The mRNA expression of FoxO family members and SIRT1 in TNBC patients of TCGA. A) The mRNA expression of FoxO members and SIRT1 between TNBC and normal tissues in TCGA (n: 135, n: 112, respectively). B) Comparisons of gene expressions between tumor samples of TNBC patients at metastasis stage (NM: non-metastasis, M: metastasis) $(n=137)$.

\section{Supplementary Files}

This is a list of supplementary files associated with this preprint. Click to download.

- Table1.tif

- Table2.tif 